\title{
Estudio Exploratorio sobre las Barreras y Apoyos a la Pertenencia en Educación Secundaria
}

\author{
Exploratory Study on Barriers and Supports to Sense of \\ Belonging in Secondary Education
}

\author{
Isabel Fernández Menor * \\ Universidad de Vigo, España
}

\begin{abstract}
Las altas tasas de fracaso y abandono escolar que preocupan al sistema educativo español no siempre han ido encaminadas con las acciones apropiadas para la reducción. Los centros educativos pueden desplegar una serie de actuaciones que modifiquen el desenganche educativo de nuestros estudiantes. En este trabajo se entiende que el sentimiento de pertenencia y la conexión escolar son predictores de fracaso y abandono, por lo que es preciso cuidar las relaciones de nuestros estudiantes, el currículum o la metodología para fortalecer una educación inclusiva. Este estudio es un micro-proyecto perteneciente al gran proyecto Redes de Innovación para la Inclusión Educativa y Social, en el que se aplica el cuestionario ¿Qué funciona para ti? Barreras y apoyos en el centro. Se realiza un análisis descriptivo y otro comparativo. Los resultados muestran sentimientos positivos en diferentes momentos y lugares del centro, ante las distintas formas de trabajo de aula y un buen sentido de pertenencia y orgullo. El análisis comparativo indica diferencias significativas en la pertenencia en cuanto al género y en todos los factores estudiados en cuanto al número de cursos en el centro y la existencia o no de dificultades sociofamiliares. En conclusión, se ofrece un estudio exploratorio que sirve como punto de partida hacia mejoras y actuaciones que nazcan desde el propio centro.
\end{abstract}

Descriptores: Enseñanza secundaria; Enseñanza para todos; Bienestar; Afectividad; Fracaso escolar.

\begin{abstract}
The high rates of school failure and dropout that concern Spanish education system have not always been aimed at appropriate actions for reduction. It is considered that schools can display a series of actions that modify the educational disengagement of our students. In this work, it is understood that the feeling of belonging and the school connection are predictors of school failure and drop out, so it is necessary to take care of the relationships of our students, the curriculum or the methodology in order to strengthen a more inclusive education. This study is a micro-project belonging to a big one: Innovation Networks for Educational and Social Inclusion project, in which the questionnaire What works for you? Barriers and supports at school is applied. A descriptive and comparative analysis is performed. The results show positive feelings at different times and places in the center, given the different forms of classroom work and a good sense of belonging and pride towards the center. The comparative analysis indicates significant differences in membership in terms of gender and in all the factors studied in terms of the number of courses in the center and the existence or not of socio-family difficulties. In conclusion, this exploratory study is offered like a starting point towards improvements and actions that are born from the school itself.
\end{abstract}

Keywords: Secondary education; Education for all; Welfare; Emotions; Academic failure.

\section{*Contacto: isfernandez@uvigo.es}

ISSN: 2254-3139

www.rinace.net/riejs/ revistas.uam.es/riejs
Recibido: $\quad 15$ de septiembre 2019

$1^{\text {a }}$ Evaluación: 16 de diciembre 2019

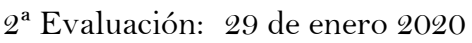

Aceptado: 2 de marzo 2020 


\section{Introducción}

El fracaso y el abandono escolar suponen en la actualidad un reto para el sistema educativo español, el cual ronda el $18 \%$ de estudiantes que dejan tempranamente sus estudios. De este modo, nos encontramos a la cola de Europa, siendo superados únicamente por Malta. Las variables, factores o indicadores de este fenómeno han sido ampliamente estudiados, no obstante, todavía no se ha conseguido reducir esta elevada tasa. La adolescencia es la etapa vital en la que se desarrollan con mayor asiduidad estos procesos de escape, coincidente con la Educación Secundaria Obligatoria (en adelante ESO). El cambio de etapa educativa, el deseo de autonomía por parte de los jóvenes, la búsqueda de la identidad o la importancia que cobran las relaciones con iguales ante las relaciones familiares son solo algunos de los rasgos definitorios de esta conflictiva fase vital. En el caso de los centros educativos, los jóvenes transitan por un sistema propedéutico con dificultades de atención a la diversidad, mínima formación docente, escasa atención a las características individuales y focalizado estrictamente en los resultados (Fielding, 2011).

En el momento en que nuestros adolescentes emprenden este camino de desenganche académico, la exclusión se hace latente. El cuidado y apoyo a los estudiantes por parte de las instituciones educativas ha de ser la hoja de ruta de nuestro sistema (Hargreaves, Earl y Ryan, 1996), entendiendo que las comunidades precisan que sus miembros encuentren respuesta a sus necesidades, se sientan integrados, existan relaciones de influencia y el sentido de conexión sea elevado (McMillan y Chavis, 1986).

El desenganche escolar es el hilo conductor de este texto, debido a que las investigaciones lo sitúan como un relevante predictor de fracaso y abandono escolar. Se trata de un concepto multidimensional que se hace visible a través del absentismo, una pobre conexión escolar o la expresión de conductas disruptivas. En esta línea, cuando hablamos de inclusión educativa, como se ha señalado reiteradamente, no solo podemos focalizarlo en dificultades del aprendizaje, alumnado con Necesidades Específicas de Apoyo Educativo o estudiantes extranjeros, sino que la inclusión es un derecho para todo el estudiantado y un deber para nuestro sistema.

Contando con la inclusión educativa como telón de fondo, se abordan aquí los procesos de enganche y desenganche escolar y la influencia que sobre estos conceptos tiene el sentimiento de pertenencia y la conexión escolar. En este artículo se presentan los resultados de la parte cuantitativa de un micro-proyecto de investigación perteneciente al proyecto nacional "Redes de Innovación para la Inclusión Educativa y Social". Este microproyecto ha sido bautizado con el nombre "En conexión", el cual resume a la perfección el cometido del mismo: conocer el sentimiento de pertenencia de nuestros estudiantes y realizar propuestas de mejora sobre el mismo.

\section{Enganche escolar, sentido de pertenencia y conexión escolar}

Desenganche y desconexión escolar son dos fenómenos que aluden de forma clara a la exclusión educativa y social del alumnado. No obstante, esta cuestión no ha sido abordada con la intensidad que merece, por lo que se estima pertinente la vinculación de los conceptos inclusión y conexión escolar. Tal y como indica Terigi (2014), el análisis de esta 
conexión es necesaria para construir trayectorias escolares que no excluyan a los estudiantes.

Los "desenganchados" son estudiantes que se sitúan en zona de riesgo (García, 2015), ya que personalmente no encuentran su lugar a pesar de que a nivel legal si formen parte del centro educativo. Es de vital importancia descubrir qué factores pueden mediar este proceso, siendo la literatura recurrente en señalar una serie de elementos tales como la inflexibilidad curricular, la desatención de las relaciones (docente-discente o discentediscente), una metodología de enseñanza-aprendizaje ancestral y una exclusiva atención a los resultados en desfavor del proceso.

Si hablar de desenganche es hablar de desconexión, hablar de enganche escolar implica compromiso, a todos los niveles, con la institución escolar. El enganche comprende la implicación en el propio proceso de aprendizaje, la participación, la adquisición de un sentimiento de pertenencia positivo hacia el centro o unas sólidas relaciones entre los diferentes miembros (González González, 2015).

El enganche escolar se compone de tres elementos que permiten avanzar en la conceptualización del término. Así, existe un factor comportamental visible en la atención que el estudiante pone en el docente, la asistencia al centro, la realización de preguntas o la entrega de trabajos, entre otros. En este sentido, el factor comportamental está vinculado con la participación de los estudiantes en el centro. El segundo factor es de carácter emocional y se vincula con la identificación del alumnado con su institución. Este tipo de enganche se manifiesta a través de las relaciones interpersonales, los sentimientos de pertenencia al centro o el estado anímico. El tercer factor es de tipo cognitivo y se refleja en el uso efectivo de estrategias y resolución óptima de problemas, una buena tolerancia al fracaso y la capacidad autorreguladora del aprendizaje (Mahatmya et al., 2012).

El cuadro 1 muestra comportamientos previsibles en función del objeto y dominio del enganche escolar y que nos permite perfilar en distintos ámbitos algunas manifestaciones.

Cuadro 1. El desenganche académico en función del objetivo y dominio de enganche

\begin{tabular}{lccc}
\hline & \multicolumn{3}{c}{ DomINIOS DEL ENGANCHE } \\
\cline { 2 - 4 } $\begin{array}{c}\text { Sistema } \\
\text { educativo }\end{array}$ & Abandono & Emocional & Cognitivo \\
\hline $\begin{array}{c}\text { Centro } \\
\text { educativo }\end{array}$ & Absentismo & $\begin{array}{c}\text { Bajación de la } \\
\text { educación }\end{array}$ & $\begin{array}{c}\text { La educación es vista } \\
\text { como algo irro el }\end{array}$ \\
\hline Aula & Faltar a clases & $\begin{array}{c}\text { Bajo interés y } \\
\text { aburrimiento }\end{array}$ & $\begin{array}{c}\text { Poca disposición hacia el } \\
\text { trabajo }\end{array}$ \\
\hline Contenido & $\begin{array}{c}\text { Comportamientos } \\
\text { disruptivos }\end{array}$ & Ansiedad & $\begin{array}{c}\text { Escasa autorregulación } \\
\text { del aprendizaje }\end{array}$ \\
\hline Fuente:
\end{tabular}

Fuente: Elaboración propia a partir de Hancock y Zubrick (2015).

El enganche escolar funciona a diferentes niveles, pudiendo encontrarnos estudiantes con un gran rendimiento académico, pero bajo enganche personal con la institución y, en el extremo opuesto, estudiantes cuyas relaciones son fructuosas pero su nivel curricular y de enganche con el aprendizaje es bajo. En este sentido, Hancock y Zubrick (2015) indican que el desenganche suele asociarse a un pobre rendimiento académico y que estas relaciones se fraguan con el tiempo resultando ser recíprocas y cíclicas. 
Ante esta desvinculación que sufren algunos estudiantes, el sistema en general y el centro en particular, tiende a culpabilizar exclusivamente al alumnado, omitiéndose las diferentes variables que determinaron esta situación. Siendo múltiples y variados los contextos influyentes, existe una ineludible responsabilidad por parte del estudiante con su propia educación, pero esta responsabilidad es compartida con el centro, que debe dar respuesta a las necesidades, inquietudes y problemáticas que puede presentar su alumnado. Asimismo, la situación social, familiar o el contexto de procedencia de cada individuo es único y las oportunidades que le son brindadas son diferentes, lo que revierte sobre el enganche o desenganche del sujeto.

A raíz de esta situación, la investigación en este campo se ha focalizado en dos vertientes, una sobre factores de riesgo que conducen al desenganche (Hancock y Zubrick, 2015; Thompson et al., 2006) y otra sobre las consecuencias de la desafección (Bond et al., 2007).

Dentro del denominado enganche emocional, tratado con anterioridad, se encuentra el sentido de pertenencia, como el sentimiento de formar parte de algo. El sistema educativo ha olvidado por completo este concepto, descuidando el bienestar emocional de sus estudiantes. Una de las primeras estudiosas sobre la pertenencia fue Carol Goodenow, quien definió el término como el grado en que los estudiantes se sienten personalmente aceptados, respetados, incluidos y apoyados por los demás en el ambiente escolar (Goodenow, 1993). Posteriormente, Baumeister y Leary (1995) comprendieron que la falta de pertenencia, como elemento de la motivación humana, desencadena problemas de salud metal, tales como estrés o psicopatías. Asimismo, la pertenencia a las diferentes comunidades (familiares, escolares, sociales, culturales...) es un factor de protección ante las mencionadas problemáticas (Resnick et al., 1997). Otro autor destacado en el campo de la pertenencia es Finn (1989), que desarrolla el modelo de identificación-participación. Este modelo se basa en la premisa de que cuanto mayor es la participación, mayores son las posibilidades de éxito académico y también de identificarse con el centro educativo; al contrario, una baja participación repercutiría en el rendimiento escolar y provocaría el abandono. En esta línea, la participación se muestra a través de la atención, de la realización de cuestiones o la participación en tareas extracurriculares; la identificación se refleja en los sentimientos de formar parte de la comunidad. La trayectoria más frecuente sería encontrarnos con alumnado con una buena valoración de la educación que se siente parte del centro, pero esto no siempre es así. Existen estudiantes participativos a nivel académico que no acaban de encontrar su lugar a nivel social y, también, alumnado con unas grandes dotes sociales pero un rendimiento bajo. Por este motivo, el modelo de Finn ha sido modificado en diferentes ocasiones: Appleton, Christenson y Furlong (2008) indicaron que los elementos que deberían componer el modelo son el académico, el comportamental, el cognitivo y el afectivo. Por su parte, Skinner y otros (2008) entendían que debía estar compuesto por únicamente dos componentes, el comportamental y el emocional. Por otro lado, Martin (2007) entendía que la división debía realizarse en función a la cognición y comportamientos adaptativos o no adaptativos.

Los resultados no son el único elemento mediador, sino que existen diferentes variables influyentes, tales como las características del centro, las relaciones con los docentes, las relaciones con iguales, el ambiente de aula, el currículo y las necesidades particulares de cada estudiante.

En relación con las características del centro, cuestiones como el tamaño, la configuración escolar o la situación geográfica son clave (Anderman, 2002). Así, los centros con menor 
tamaño, los que abarcan más etapas educativas y los centros de entorno rural favorecen en mayor medida la pertenencia, ya que tienen más posibilidades de conocer a todos los integrantes y transitan por el mismo durante más tiempo (Eisner, 2001; Freeman, Hughes y Anderman, 2001).

Las relaciones con los docentes impactan sobremanera en la experiencia educativa de los estudiantes. De este modo, el apoyo que el profesorado ofrece a los estudiantes fortalece su seguridad (Luckner y Pianta, 2011), mientras que los conflictos empobrecen la motivación y el rendimiento (Canales y Peña, 2014). Existen también relaciones de dependencia docente-discente que se van perdiendo a medida que se avanza en el sistema, lo que implica unas relaciones menos próximas afectando a la pertenencia.

En un momento como la adolescencia, en el cual las relaciones se vuelven significativas, la amistad y el rechazo son cuestiones relevantes para la pertenencia. Resulta evidente que las relaciones de amistad fortalecen la pertenencia, aumentando la adaptación, el bienestar y el ajuste social. En el otro extremo, el rechazo produce baja autoestima y una reducción considerable de la participación (Canales y Peña, 2014).

Otra variable destacada es el ambiente de clase. El clima que se promueva en el aula determina el ajuste comportamental y emocional del alumnado (Shochet y Smith, 2014). Por su parte, Loukas, Suzuki y Horton (2006) entienden que los sentimientos de pertenencia y el ambiente de aula se encuentran vinculados en función a cuatro variables: fricción, cohesión, competición y satisfacción. Un ambiente de cohesión y satisfacción promueve la pertenencia, mientras que un ambiente competitivo y con tensión la disminuye.

El currículo propuesto también interviene en la pertenencia. Su estructuración en bloques le confiere un carácter parcelario y academicista que facilita el desarrollo de metodologías pasivas que no solventan las necesidades particulares de nuestros alumnos y alumnas.

Finalmente, las características personales de cada individuo son cruciales en la pertenencia, ya que la necesidad de pertenecer no es la misma para todos. La autonomía, la competencia, la percepción de control o la forma en la que se despliegan las estrategias de resolución de problemas son cuestiones imprescindibles en nuestros sentimientos de pertenecer.

\section{Método}

\section{Enfoque metodológico}

El enfoque metodológico empleado en el ámbito del estudio que se presenta es de tipo cuantitativo ya que se trata de un problema concreto y delimitado, buscándose la afirmación o rechazo de una serie de hipótesis de trabajo. Para ello se emplean instrumentos de medición cuyos resultados son posteriormente analizados mediante métodos estadísticos. Su carácter más objetivo permite extraer una serie de generalizaciones posiblemente aplicables al contexto social (Hernández, Fernández y Baptista, 2010). En este sentido, el objeto de este estudio es analizar el sentido de pertenencia del alumnado de educación secundaria de los centros públicos de Pontevedra ciudad con respecto a su centro. 


\section{Variables}

En este trabajo, las variables dependientes se corresponden con los factores extraídos a través del análisis factorial del cuestionario; en este caso se trata de: "dinámicas escolares colectivas", "confort y seguridad emocional", "actividades académicas individuales" y "sentimiento de pertenencia".

Por otro lado, las variables independientes son las destacadas como variables sociodemográficas en el cuestionario y con las que se ha decidido realizar el análisis comparativo: género, número de cursos que los estudiantes llevan en el centro y, en último lugar, la existencia o no de circunstancias socio-familiares adversas. En cuanto al género, se dan tres opciones de respuesta: hombre, mujer u otro. En relación con el número de cursos, se refiere a la cantidad de años que el estudiante lleva en el centro, ya que puede ser nuevo/a, seguir la trayectoria normal o estar repitiendo. En cuanto a las circunstancias socio-familiares adversas, se refiere a una situación social o familiar complicada: problemáticas económicas, desarrollo en zonas sociales desfavorables, familias desestructuradas, etc.

\section{Población, muestra y muestreo}

El universo de este estudio son los centros educativos públicos de Educación Secundaria de la ciudad de Pontevedra (Galicia, España). El muestro es de tipo no probabilístico, "donde las unidades muestrales son elegidas mediante criterios subjetivos y no mediante una selección aleatoria” (Boza, Pérez y de León, 2016, p.14). Se trata de un muestreo intencional en el que interesan los estudiantes del $2^{\circ}$ curso de ESO, por ser un momento crucial dada la edad de los estudiantes, en el que comienzan muchos procesos de enganche o desenganche escolar, como se ha venido tratando en la revisión de literatura de este artículo. En total, el universo está compuesto por los siete centros públicos existentes en la ciudad de Pontevedra, por ser un número reducido y de fácil acceso. La muestra de estudiantes participantes es de 331, desglosándose como se muestra en el cuadro 2.

Cuadro 2. Número de participantes por centro en la primera fase del estudio

\begin{tabular}{cc}
\hline CENTRO & N $^{\circ}$ DE PARTICIPANTES \\
\hline${\text { Centro }{ }^{\circ} 1}^{\circ}$ & 19 \\
Centro $^{\circ} \mathcal{Q}$ & 57 \\
Centro $^{\circ} 3$ & 39 \\
Centro $^{\circ} 4$ & 59 \\
Centro $^{\circ} 5$ & 37 \\
Centro n $^{\circ} 6$ & 58 \\
Centro n $^{\circ} 7$ & 62 \\
\hline Total & 331 \\
\hline
\end{tabular}

Fuente: Elaboración propia.

Estos centros están situados en zona urbana o periurbana y abarcan las etapas educativas de ESO, Bachillerato y Formación Profesional. El número de alumnos y alumnas oscila entre los 600 y los 1100 y el número de docentes entre 60 y 107. Las diferencias en el número de estudiantes de los centros vienen dadas por su situación, ya que dos de las instituciones están situadas a escasos metros de distancia, por lo que el número de estudiantes es menor. Tras el contacto con los siete centros que componen el estudio, todos aceptan la participación y se da comienzo a la aplicación del cuestionario “QQué funciona para ti? Barreras y apoyos a la pertenencia en los centros” (Porter et al., 2010). 


\section{Instrumentos de obtención de información}

La aplicación del cuestionario ha sido extensiva en cuanto a que implica a todos los centros públicos de educación secundaria de la ciudad de Pontevedra. El instrumento empleado ha sido el cuestionario "What works for you? Barriers and supports at school", diseñado por la profesora Jill Porter de la Universidad de Reading (Reino Unido). La versión original es en inglés y su soporte es online. Consta de tres bloques de preguntas, siendo 38 el número total. El primer bloque trata los sentimientos de los estudiantes en diferentes momentos y lugares; el segundo, versa sobre diferentes formas de trabajar en el aula y, finalmente, el tercero trata sobre los sentimientos de los estudiantes en el instituto.

No obstante, este cuestionario ha sido traducido y adaptado en su aplicación al contexto gallego. Una vez traducido, el cuestionario se aplicó a 50 estudiantes de $2^{\circ}$ curso de ESO de un instituto de características similares a los de la muestra. Tras la realización de esta prueba piloto se modifican determinados aspectos del cuestionario:

- Cambio en el número de preguntas, de 38 a 46.

- Se añaden cuestiones sociodemográficas.

- Se añade una pregunta sobre relaciones con iguales.

- Se ajustan cuestiones de difícil comprensión.

Esta prueba piloto, así como el análisis factorial que posteriormente se presenta, se realizan con el objetivo de ofrecer validez y fiabilidad al instrumento traducido. Tras las adaptaciones, se procede a la aplicación del instrumento final, el cuestionario "¿Qué funciona para ti? Barreras y apoyos en el centro". Atendiendo a las peculiaridades del contexto educativo y social de Pontevedra, el cuestionario online se ofreció en dos idiomas, gallego y castellano.

\section{Trabajo de campo}

El trabajo que aquí se presenta es el resultado de un proyecto más amplio, denominado "En conexión" perteneciente al gran proyecto "Redes de Innovación para la Inclusión Educativa y Social”. De este modo, el proyecto "En conexión" es de corte mixto, ya que la primera de las fases (presentada en este trabajo) es de tipo cuantitativo a pesar de que el resto es de corte cualitativo. Asimismo, esta primera fase es extensiva, mientras que el resto son intensivas por haber sido desarrolladas como un estudio de caso. A continuación, se presenta la figura 1, que esquematiza el proyecto general para facilitar la comprensión del mismo.

\section{Análisis de datos}

El análisis de datos ha sido doble: análisis descriptivo y análisis comparativo. Para el análisis descriptivo se han tenido en cuenta cada una de las variables de los tres bloques del cuestionario, trabajando con un total de veinticuatro variables, de las cuales seis se corresponden con el primer bloque, seis con el segundo y doce con el tercero. Estas variables se muestran en el cuadro 3. Su análisis ha sido realizado mediante el software estadístico Statistical Package for the Social Sciences en su versión 25 (en adelante SPSS). 


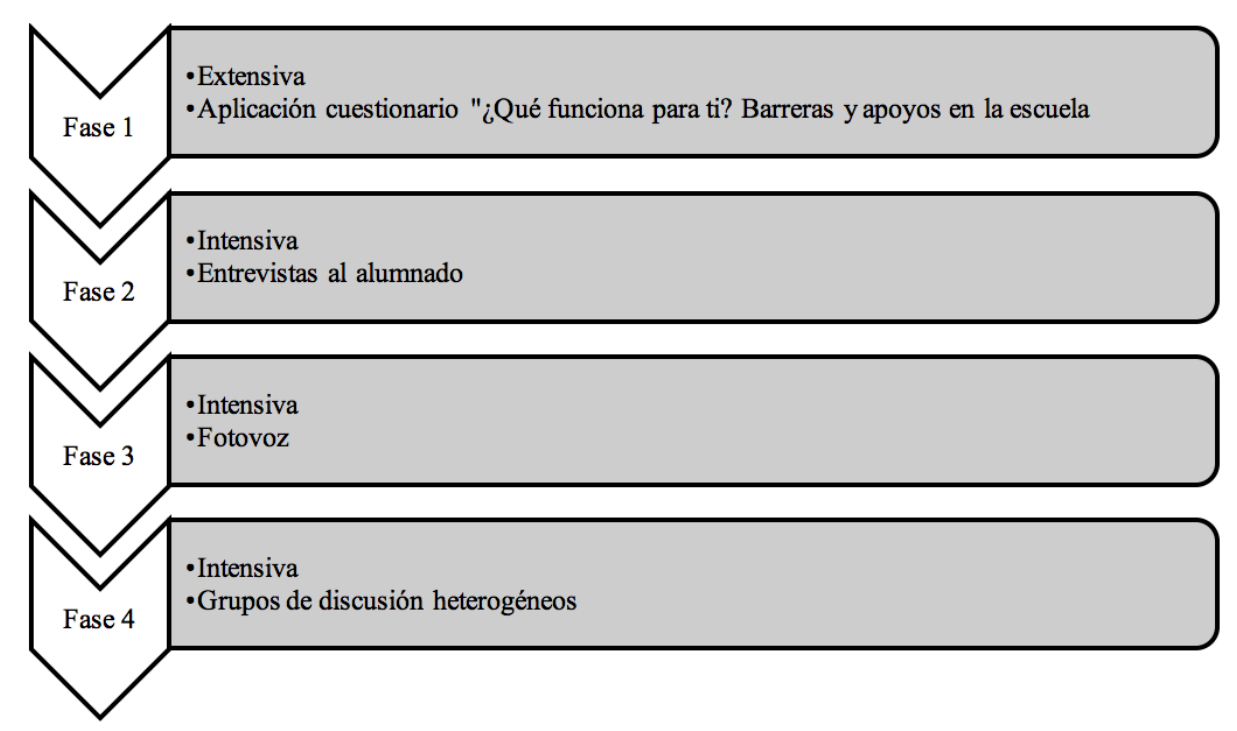

Figura 1. Fases del proyecto "En conexión"

Fuente: Elaboración propia.

Para ofrecer validez y consistencia interna al cuestionario se ha hecho un análisis factorial, para el que nuevamente es útil el software SPSS. Para ello, se ha comprobado la interrelación de los ítems a través de la prueba Kaiser-Meyer-Olkin y la prueba de esferacidad de Bartlett. Para conocer la normalidad de la muestra se emplea la prueba Kolmogorov-Smirnov. La extracción de los factores se realiza por medio de la factorización de ejes principales y la prueba Oblimin directo para la rotación factorial. Finalmente, la consistencia interna de los factores es realizada con Alpha de Cronbach.

Los factores obtenidos con la dimensionalidad del instrumento son los siguientes: "dinámicas escolares colectivas", "confort y seguridad emocional", "actividades académicas individuales" y "sentimiento de pertenencia". Estos factores están constituidos por las variables que se muestran en el cuadro 4.

Una vez identificados los factores, tiene lugar el análisis comparativo, en el que se realiza un estudio de contraste para el género, el número de cursos y la existencia o no de circunstancias socio-familiares adversas. Tras el análisis de normalidad de las distribuciones de respuestas y la homogeneidad de las varianzas dentro de los grupos, se comprueba que la muestra no es normal, por lo que se emplean pruebas no paramétricas (Kruskall-Wallis o prueba de la mediana de Mood). Para el análisis comparativo del género, se empleó la mediana de Mood para los factores "dinámicas escolares colectivas" y "confort y seguridad emocional”; por otro lado, se empleó la prueba Kruskall-Wallis para los factores "actividades académicas individuales" y "sentimiento de pertenencia". Para el análisis de los cursos que los estudiantes llevan en el centro, se aplica nuevamente la prueba de Kruskall-Wallis. En último lugar, para el análisis de la existencia o no de circunstancias socio-familiares adversas se emplea la prueba U de Mann Whitney, ya que se trata de una pregunta dicotómica. 
Cuadro 3. Nomenclatura de las variables en función del bloque

\begin{tabular}{|c|c|c|}
\hline BLOQUE & $\begin{array}{c}\text { NOMENCLATURA } \\
\text { ABREVIADA } \\
\end{array}$ & NOMENCLATURA COMPLETA \\
\hline \multirow{6}{*}{$\begin{array}{l}\text { Sentimientos en } \\
\text { diferentes } \\
\text { momentos y } \\
\text { lugares }\end{array}$} & SMLCLAS & Sentimientos durante las clases \\
\hline & SMLREC & Sentimientos durante el recreo \\
\hline & SMLCOMI & Sentimientos durante la comida \\
\hline & SMLCAMB & Sentimientos en los cambios de clase \\
\hline & SMLEVEN & Sentimientos en eventos especiales \\
\hline & SMLEXC & Sentimientos en excursiones y viajes escolares \\
\hline \multirow{6}{*}{$\begin{array}{l}\text { Sentimientos } \\
\text { según las } \\
\text { diferentes formas } \\
\text { de trabajar en el } \\
\text { aula }\end{array}$} & STACLASTIP & Sentimientos en una clase típica \\
\hline & STAIND & Sentimientos trabajando individualmente \\
\hline & STACLASPRAC & Sentimientos en las clases prácticas \\
\hline & STADEP & Sentimientos en clases de deporte \\
\hline & STAEXA & Sentimientos ante exámenes \\
\hline & STADEB & Sentimientos ante deberes \\
\hline \multirow{12}{*}{$\begin{array}{l}\text { Sentimientos en el } \\
\text { instituto }\end{array}$} & SIPARTE & Sentirse parte del centro \\
\hline & SIAMABLE & La gente es amable conmigo \\
\hline & SIRESP & Soy tratado/a con respeto \\
\hline & SIYOMISM & Puedo ser yo mismo/a \\
\hline & SIPROF & Los profesores/as me respetan \\
\hline & SIORGULLO & Estoy orgulloso/a de pertenecer a este centro \\
\hline & SISEGCLAS & Me siento seguro/a durante las clases \\
\hline & SISEGDES & Me siento seguro/a durante los descansos \\
\hline & SIAMIGOS & Puedo estar con mis amistades en este centro \\
\hline & SISOLO & Puedo pasar tiempo solo/a si quiero \\
\hline & SIFUERA & $\begin{array}{l}\text { Fuera del centro, no encuentro difícil hacer lo } \\
\text { que quiero hacer }\end{array}$ \\
\hline & SIASIST & $\begin{array}{l}\text { Existen o no circunstancias para mi asistencia al } \\
\text { centro }\end{array}$ \\
\hline
\end{tabular}

Fuente: Elaboración propia.

Cuadro 4. Factores y variables que los componen

\begin{tabular}{ll}
\hline \multicolumn{1}{c}{ FACTORES } & \multicolumn{1}{c}{ VARIABLES } \\
\hline Dinámicas escolares colectivas & $\begin{array}{l}\text { SMLCLAS, SMLREC, SMLCOMI, } \\
\text { SMLEVEN, SMLEXC, SMLCLASPRAC }\end{array}$ \\
\hline Confort y seguridad emocional & $\begin{array}{l}\text { SIAMABLE, SIRESP, SIYOMISM, SIPROF, } \\
\text { SISEGCLAS }\end{array}$ \\
\hline Actividades académicas individuales & $\begin{array}{l}\text { STACLASTIP, STAIND, STAEXA, } \\
\text { STADEB }\end{array}$ \\
\hline Sentimiento de pertenencia & $\begin{array}{l}\text { SIPARTE, SIORGULLO, SIFUERA, } \\
\text { SIASIST }\end{array}$ \\
\hline
\end{tabular}

Fuente: Elaboración propia.

\section{Resultados}

\subsection{Análisis descriptivo}

Para mostrar de forma descriptiva los resultados del cuestionario, se presenta la media, mediana y moda de los ítems de cada bloque, donde el primero de los bloques hace referencia a los sentimientos en diferentes momentos y lugares, el segundo a los sentimientos según las diferentes formas de trabajar en el aula y, finalmente, el tercero, a los sentimientos en el instituto.

Los resultados obtenidos en relación con el primer bloque, sentimientos en los diferentes momentos y lugares, son los recogidos en el cuadro 5. La forma de respuesta a estas cuestiones es tipo Likert de uno a seis, donde uno significa "muy bien” y seis "muy mal”, 
las medias aritméticas que tienden a uno son más favorables que las que tienden a seis. Los resultados obtenidos son positivos debido a que la media es inferior a tres en todos los casos, siendo este número el divisor entre los sentimientos positivos y los negativos. El valor que más se repite, es decir, la moda, es uno en todos los casos a excepción de los sentimientos en las clases, que es tres. Se ha obtenido una desviación típica baja todos los casos por lo que los datos tienden a agruparse en torno a la media aritmética.

Cuadro 5. Número de sujetos, media, moda y desviación típica obtenidas en los ítems sobre diferentes momentos y lugares en el centro

\begin{tabular}{lcccc}
\hline & N & MEDIA & MODA & DESV. TíPICA \\
\hline Sentimientos durante las clases & 328 & 2,66 & 3 & 1,10 \\
Sentimientos durante el recreo & 327 & 1,61 & 1 & 0,91 \\
Sentimientos durante la comida & 104 & 2,12 & 1 & 1,27 \\
Sentimientos en los cambios de clase & 329 & 2,18 & 1 & 1,09 \\
Sentimientos durante eventos del centro & 317 & 1,97 & 1 & 1,22 \\
Sentimientos durante las excursiones & 312 & 1,75 & 1 & 1,13 \\
\hline
\end{tabular}

Fuente: Elaboración propia.

En la figura 2 se presentan los resultados agrupados en sentimientos positivos y negativos. Los positivos hacen referencia a la suma de los porcentajes obtenidos con las respuestas "muy bien", "bastante bien" y "bien". Los negativos se componen de la suma de los porcentajes obtenidos con las respuestas "regular", "mal” y "muy mal":

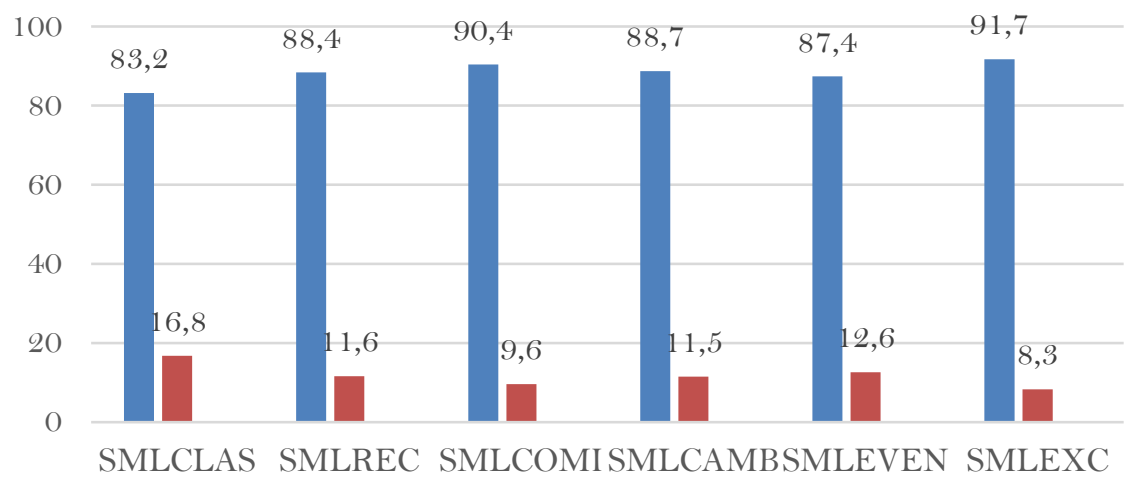

口 Sentimientos positivos $\square$ Sentimientos negativos

Figura 2. Porcentaje de sentimientos positivos y negativos por ítem Fuente: Elaboración propia.

Tal y como muestra la figura anterior, un 91,7\% de los estudiantes consideran positivos sus sentimientos durante las excursiones, siendo el ítem más valorado, seguido por los sentimientos durante la comida (90,4\%), los cambios de clase $(88,5 \%)$, el recreo $(88,4 \%)$, los eventos especiales $(87,4 \%)$ y, en último lugar, las clases $(83,2 \%)$. En contraposición, el $16,8 \%$ de los estudiantes tienen sentimientos negativos durante las clases, seguido de los eventos $(12,6 \%)$, el recreo $(11,6 \%)$, los cambios de clase $(11,5 \%)$, la comida $(9,6 \%)$ y, por último, las excursiones $(8,3 \%)$.

El segundo bloque, relativo a las diferentes formas de trabajar en el aula, muestra los resultados presentados en el cuadro 6. En este caso, los resultados varían con respecto al bloque anterior. Las medias tienden a tres en los dos primeros ítems y lo superan en los dos últimos. La moda indica que los sentimientos hacia las clases típicas, el trabajo 
individual y los deberes es bueno, siendo regular en el caso de los exámenes. No obstante, las clases prácticas y los sentimientos durante la realización de deporte son muy buenos. Nuevamente, una baja desviación típica nos muestra la agrupación de las respuestas en torno a la media aritmética.

Cuadro 6. Número de sujetos, media, moda y desviación típica obtenidas en los ítems relativos a los sentimientos ante las diferentes formas de trabajar en el aula

\begin{tabular}{lcccc}
\hline & N & MEDIA & MODA & DESV. TíPICA \\
\hline Sentimientos en una clase típica (magistral) & 327 & 2,76 & 3 & 1,03 \\
Sentimientos ante el trabajo individual & 325 & 2,65 & 3 & 1,20 \\
Sentimientos en las clases prácticas & 327 & 2,02 & 1 & 1,07 \\
Sentimientos mientras se realiza deporte & 329 & 2,05 & 1 & 1,23 \\
Sentimientos ante los exámenes & 330 & 3,65 & 4 & 1,25 \\
Sentimientos ante los deberes & 328 & 3,45 & 3 & 1,20 \\
\hline
\end{tabular}

Fuente: Elaboración propia.

La figura 3 muestra los resultados positivos y negativos sobre los sentimientos ante las diferentes formas de trabajar en el aula:

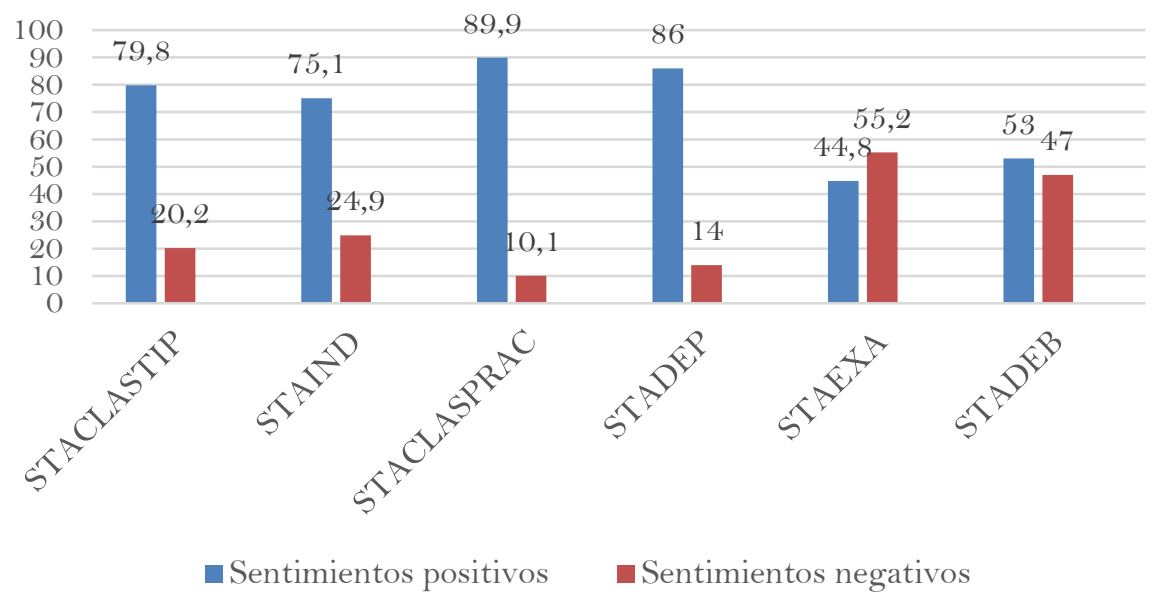

Figura 3. Porcentaje de sentimientos positivos y negativos por ítem Fuente: Elaboración propia.

Como se puede observar, las clases prácticas alcanzan los sentimientos más favorables, con un $89,9 \%$ de estudiantes que los identifican como muy buenos, bastante buenos o buenos. A este porcentaje le siguen los sentimientos durante las clases donde se practica deporte (86\%), las clases típicas (79,8\%), el trabajo individual (75,1\%), los deberes $(53 \%)$ y, en última posición, los exámenes $(44,8 \%)$. Es congruente que el porcentaje mayor en sentimientos negativos se alcance en los exámenes, con un $55,2 \%$, seguido de los deberes (47\%), el trabajo individual $(24,9 \%)$ y las clases típicas $(20,2 \%)$. Estos porcentajes muestran la inclinación hacia el trabajo práctico y el rechazo hacia la evaluación.

Con relación al bloque de sentimientos en el instituto, los resultados son los reflejados en el cuadro 7. En esta ocasión, la respuesta a los diez primeros ítems se formula en escala tipo Likert de uno a cinco, significando uno "nunca" y cinco "siempre". Por el contrario, los dos últimos ítems también se formulan en escala tipo Likert, pero en orden de uno a seis; en este caso, uno significa "sí, siempre" y seis "nunca". En esta línea, la media aritmética resulta positiva en todos los casos, tendiendo hacia las opciones "normalmente" 
o "siempre", lo cual indica que los sentimientos del alumnado para estos ítems son buenos. En el caso de las dos últimas variables, la tendencia hacia el cinco indica que los estudiantes "casi nunca" tienen dificultades para hacer las cosas que desean realizar, ni existen circunstancias que les obliguen a faltar al centro.

La moda, situada en torno al cinco, nos indica que la mayor parte del alumnado considera que se siente parte del centro, que la gente del instituto es amable con ellos/as, que son tratados con tanto respeto como el resto de estudiantes, que pueden ser ellos/as mismos/as en el centro, que el profesorado les respeta, se sienten orgullosos de pertenecer al instituto, se sienten seguros/as durante los descansos y las clases, pueden estar con sus amistades en muchos lugares del centro y, finalmente, pueden pasar tiempo solos/as si así lo desean. Además, los estudiantes "casi nunca" tienen dificultades para hacer lo que desean hacer. Finalmente, en cuanto a las circunstancias que les obliguen a faltar, el valor más repetido es "nunca", lo que indica que no existen circunstancias que obliguen a faltar al alumnado. La desviación típica nos indica que los datos se agrupan alrededor de la media aritmética.

Cuadro 7. Número de sujetos, media, moda y desviación típica obtenidas en los ítems relativos a los sentimientos en el instituto

\begin{tabular}{lcccc}
\hline & N & MEDIA & MODA & DESV. TíPICA \\
\hline Sentirse parte del centro & 329 & 3,93 & 4 & 1,11 \\
Son amables conmigo & 328 & 4,03 & 4 & 0,69 \\
Me siento respetado & 328 & 4,22 & 5 & 0,84 \\
Puedo ser yo mismo/a & 325 & 4,22 & 5 & 1,03 \\
Los profesores/as me respetan & 326 & 4,28 & 5 & 0,82 \\
Siento orgullo por pertenecer a este centro & 328 & 4,09 & 5 & 1,07 \\
Me siento seguro/a en las clases & 326 & 4,48 & 5 & 0,77 \\
Me siento seguro/a en los descansos & 318 & 4,62 & 5 & 0,67 \\
Puedo estar con mis amistades en el centro & 324 & 4,22 & 5 & 0,90 \\
Puedo pasar tiempo solo/a si quiero & 325 & 3,63 & 5 & 1,28 \\
Fuera de centro existen dificultades para & 323 & 4,66 & 5 & 1,29 \\
hacer lo que quiero hacer & & & & 1,34 \\
Existen circunstancias que me obligan a & 325 & 4,91 & 6 & \\
faltar al centro & & & & \\
\hline
\end{tabular}

Fuente: Elaboración propia.

La figura 4 muestra la agrupación de los sentimientos positivos (suma de porcentajes de las opciones de respuesta positivas) y negativos (suma de porcentajes de las opciones de repuesta negativa) en el instituto.

La seguridad en las clases y descansos alcanza porcentajes muy satisfactorios $(92 \%$ y $94,3 \%)$. Por otro lado, el $85,6 \%$ del alumnado se siente respetado por sus docentes y el $83,9 \%$ considera que en el instituto son amables con ellos/as. El 72,2\% de los estudiantes se sienten parte de su centro y un $76,2 \%$ se sienten orgullosos/as de pertenecer al mismo. Por su parte, en las dos últimas variables de este bloque, referentes a hacer lo que se quiere hacer fuera del centro y la existencia o no de circunstancias que los obliguen a faltar (figura 5), los resultados son positivos, con un $80,8 \%$ y un $84,9 \%$ respectivamente. 


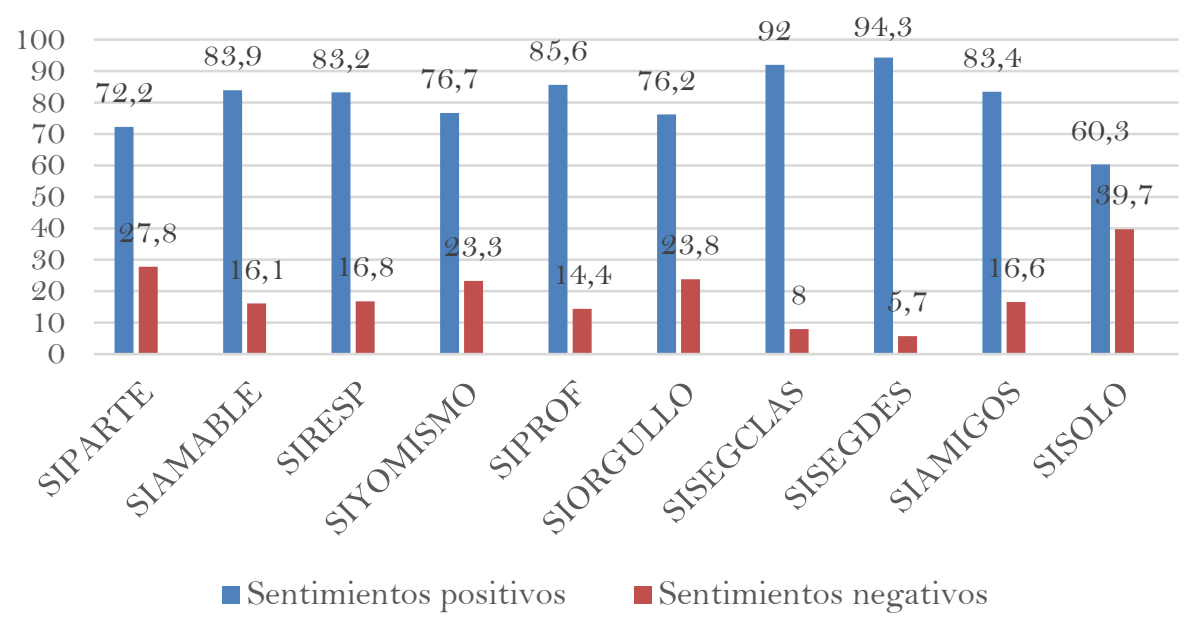

Figura 4. Porcentaje de sentimientos positivos y negativos por ítem Fuente: Elaboración propia.

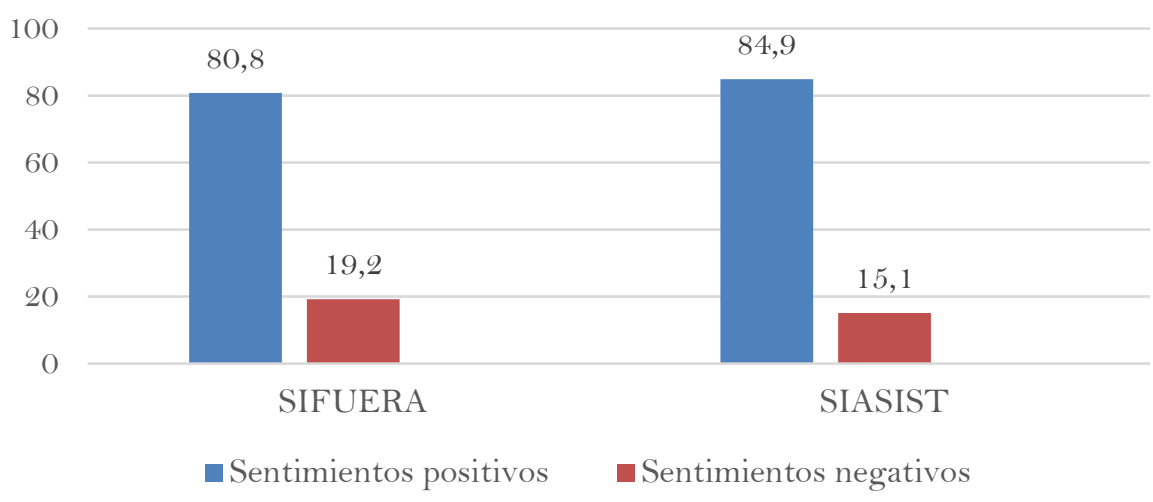

Figura 5. Porcentaje de sentimientos positivos y negativos por ítem Fuente: Elaboración propia.

\subsection{Análisis comparativo}

Para la realización de este análisis comparativo se han cruzado las tres variables sociodemográficas del cuestionario (género, número de cursos en el centro y existencia o no de circunstancias socio-familiares adversas) con las cuatro variables creadas a partir del análisis factorial efectuado (dinámicas escolares colectivas, actividades académicas de tipo individual, confort y seguridad emocional en el centro y sentimiento de pertenencia).

En los siguientes apartados figuran los resultados obtenidos en función de cada variable.

\subsubsection{Género}

En la variable género se permitía marcar tres opciones: hombre, mujer u otro. Los resultados se reflejan en el cuadro 8 .

Teniendo en cuenta una significatividad menor o igual a 0,05, se entiende que la pertenencia es significativa para el género. En el cuadro 9 se muestran el número de sujetos, la media, mediana y desviación típica de mujeres, hombres y otros, para mejorar la interpretación de los resultados. 
Cuadro 8. Grado de significatividad. Cruce de la variable "género" con las variables de los factores obtenidos

\begin{tabular}{cccc}
\hline Diná.ESCOLARES & ACTIVINDIVIDUAL & CONFORTSEGUR & PERTENENCIA \\
\hline 0,067 & 0,226 & 0,180 & 0,004 \\
\hline
\end{tabular}

Fuente: Elaboración propia.

Cuadro 9. Cantidad de sujetos, media, mediana y desviación típica para la variable significativa del género

\begin{tabular}{lcccc}
\hline & N & MEDIA & MEDIANA & DESV. TÍPICA \\
\hline Mujeres & 158 & 4,51 & 4,50 & 0,742 \\
Hombres & 152 & 4,31 & 4,50 & 0,866 \\
Otros & 6 & 3,62 & 3,62 & 0,379 \\
\hline
\end{tabular}

Fuente: Elaboración propia.

Es preciso tener en cuenta que el número de personas identificadas bajo el género "otros" es mucho más reducido (seis únicamente) que el de las personas identificadas como "hombre" o "mujer".

Siendo la variable "pertenencia" significativa, se muestra la figura 6, que permite ver que la distribución de las respuestas para la pertenencia en relación con el género es homogénea. La mediana de las mujeres y de los hombres es de 4,50 lo cual indica buenos sentimientos de pertenencia, a pesar de los tres casos atípicos que se pueden encontrar en las mujeres y de los ocho casos atípicos y un caso extremo que se detectan en los hombres. La mediana de las personas identificadas como "otros" es de 3,62, lo que indica sentimientos menos positivos que el género "hombre" o "mujer" en relación con la pertenencia.

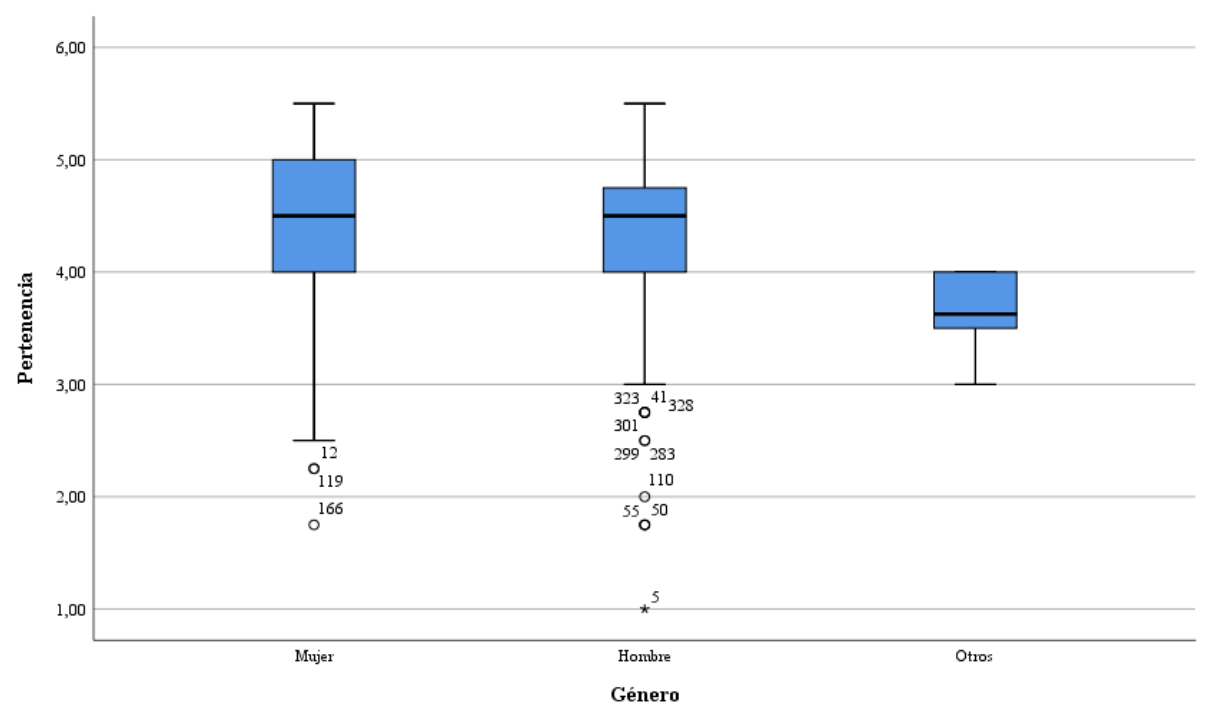

Figura 6. Gráfica sobre la pertenencia en su relación al género Fuente: Elaboración propia.

Las diferencias significativas existentes se dan entre el género "otros" y los géneros "hombre" y "mujer", como se puede observar en el cuadro 10. 
Cuadro 10. Comparativa entre grupos de género

\begin{tabular}{llcc}
\hline & & SIGNIFICATIVIDAD & SIGNIFICATIVIDAD AJUSTADA \\
\hline Mujer & Hombre & 0,081 & 0,244 \\
Mujer & Otros & 0,002 & 0,007 \\
Hombre & Otros & 0,010 & 0,030 \\
\hline
\end{tabular}

Fuente: Elaboración propia.

La figura 7 refleja las diferencias existentes entre el género "otros" con los géneros "hombre" y "mujer".

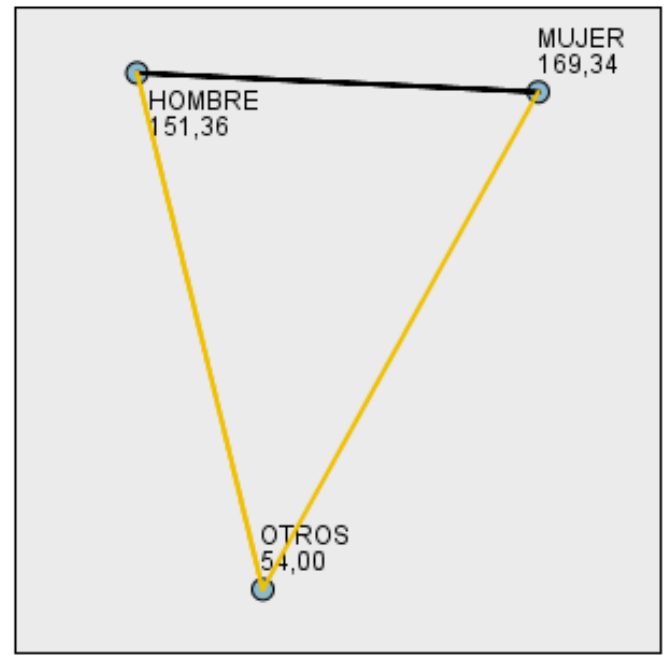

Figura 7. Comparación entre parejas de género Fuente: Elaboración propia.

Se ha decidido tener en cuenta esta diferencia significativa, a pesar de la existencia de únicamente seis sujetos amparados bajo el género "otros" debido a la intencionalidad de esta opción de respuesta en el cuestionario. Es preciso destacar que las personas que no se sienten hombre o mujer experimentan una pertenencia menor con su centro.

\subsubsection{Número de cursos en el centro}

Asumiendo que el número de cursos que los estudiantes llevan en el centro es un factor primordial en la pertenencia al centrar la muestra en el $2^{\circ}$ curso de la ESO, el alumnado podía llevar un curso en el centro si era nuevo; dos cursos si estaba en el mismo centro desde que entró en el instituto o, tres cursos si era repetidor/a.

Los resultados indican que existen diferencias significativas en función del número de cursos para las variables estudiadas, tal y como se muestra en el cuadro 11:

Cuadro 11. Grado de significatividad. Cruce de la variable sociodemográfica "número de cursos" con las variables estudiadas

\begin{tabular}{cccc}
\hline DINÁMICAS & ACTIV. & CONFORT. & PERTENENCIA \\
ESCOLARES & INDIVIDUAL & SEGUR. & PEO,000 \\
\hline 0,019 & 0,027 & 0,009 & 0.9 \\
\hline
\end{tabular}

Fuente: Elaboración propia. 
Siendo todas las variables inferiores a 0,05, son significativas. En el cuadro 12 se puede observar que el mayor número de estudiantes se sitúa en dos cursos, debido a que es la trayectoria normal de los sujetos.

Cuadro 12. Número de sujetos, media, desviación típica y mediana en función al número de cursos

\begin{tabular}{llrrrr}
\hline \multirow{2}{*}{ CURSO } & \multicolumn{1}{c}{ DeSCRIP. } & $\begin{array}{c}\text { DINÁMICAS } \\
\text { EsCOLARES }\end{array}$ & $\begin{array}{c}\text { ACTIV. } \\
\text { INDIVIDUAL }\end{array}$ & $\begin{array}{r}\text { CONFORT. } \\
\text { SEGUR. }\end{array}$ & PERTENENCIA \\
\hline \multirow{2}{*}{1} & N & 3 & 10 & 9 & 10 \\
& Media & 2,43 & 3,48 & 3,86 & 3,67 \\
& Desv. Tip. & 0,377 & 0,342 & 0,692 & 1,099 \\
& Mediana & 2,57 & 3,50 & 4,20 & 4 \\
\hline \multirow{2}{*}{2} & N & 81 & 266 & 258 & 260 \\
& Media & 1,95 & 3,07 & 4,31 & 4,52 \\
& Desv. Tip. & 0,843 & 0,847 & 0,619 & 0,731 \\
& Mediana & 1,71 & 3,00 & 4,40 & 4,75 \\
\hline \multirow{2}{*}{3} & N & 9 & 30 & 30 & 30 \\
& Media & 2,49 & 3,43 & 4,10 & 4,10 \\
& Desv. Tip. & 0,759 & 0,953 & 0,527 & 0,652 \\
& Mediana & 2,43 & 3,25 & 4,00 & 4,25 \\
\hline
\end{tabular}

Fuente: Elaboración propia.

Las figuras presentadas a continuación (figuras 8, 9, 10 y 11), se corresponden con cada una de las variables estudiadas en relación con el número de cursos en la institución.

Las dinámicas escolares ofrecen una amplia dispersión de los resultados para las personas que llevan tres cursos y reducida en los que llevan un curso. La mediana más baja se corresponde con las personas que llevan dos cursos, por lo que estas personas se sienten mejor en lo que a dinámicas escolares se refiere, a pesar de la existencia de dos casos atípicos y dos extremos; es decir, las personas que siguen la trayectoria estipulada por curso son los que mejor se sienten en las clases y cambios de clase, los recreos, durante la comida, los eventos, las excursiones y durante las clases prácticas.

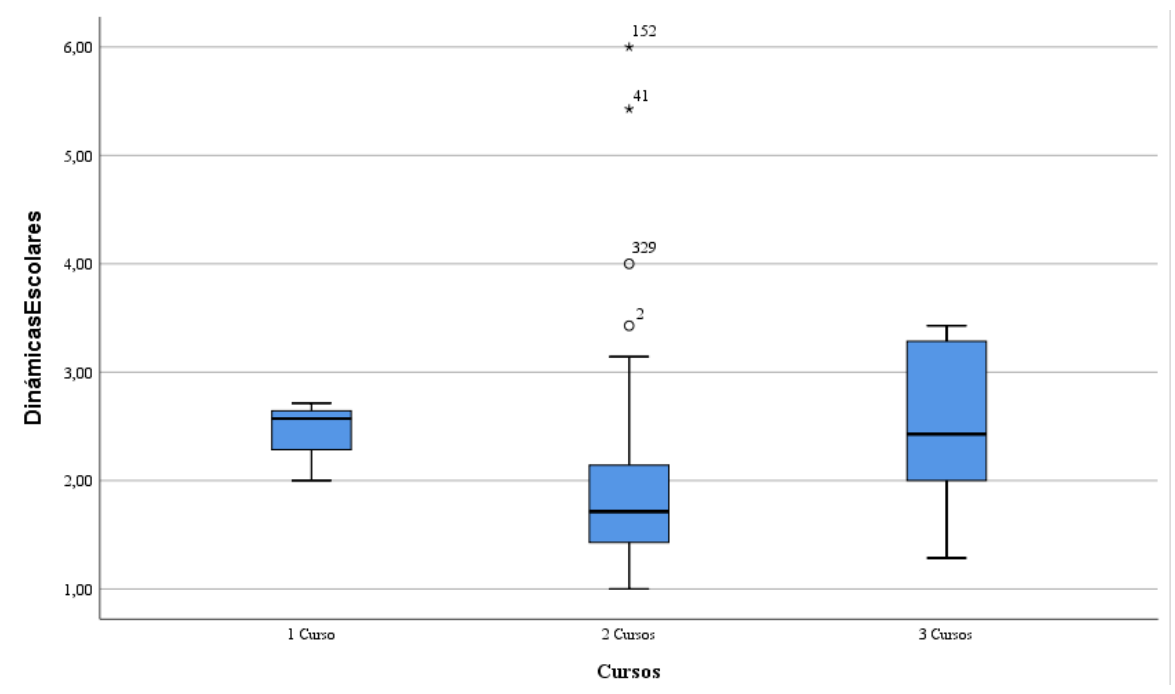

Figura 8. Dinámicas escolares en función al número de cursos en el centro Fuente: Elaboración propia. 
Con relación a las actividades de tipo individual, la distribución es más amplia en los estudiantes que llevan tres cursos en el centro, siendo, por el contrario, más homogénea en los que llevan un curso. Nuevamente, con una mediana de 3 para los estudiantes que llevan dos cursos, son los que mejor se sienten en las clases típicas, trabajando individualmente y durante la realización de exámenes y deberes.

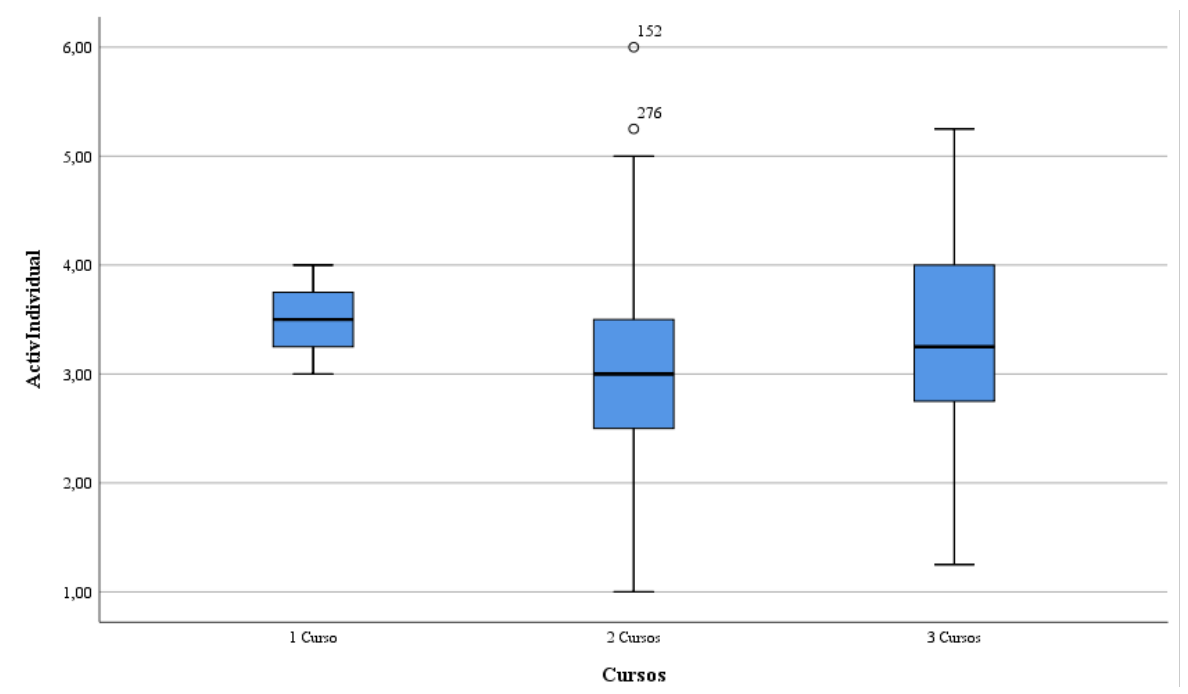

Figura 9. Actividad individual en función al número de cursos en el centro Fuente: Elaboración propia.

Confort y seguridad es un factor que presenta una distribución homogénea. Con una mediana de 4,40, los estudiantes que llevan dos cursos son los que consideran en mayor medida que las personas del centro son amables, que son tratados/as con respeto por sus profesores/as y compañeros/as, que pueden ser ellos/as mismos/as y se sienten seguros/as durante las clases.

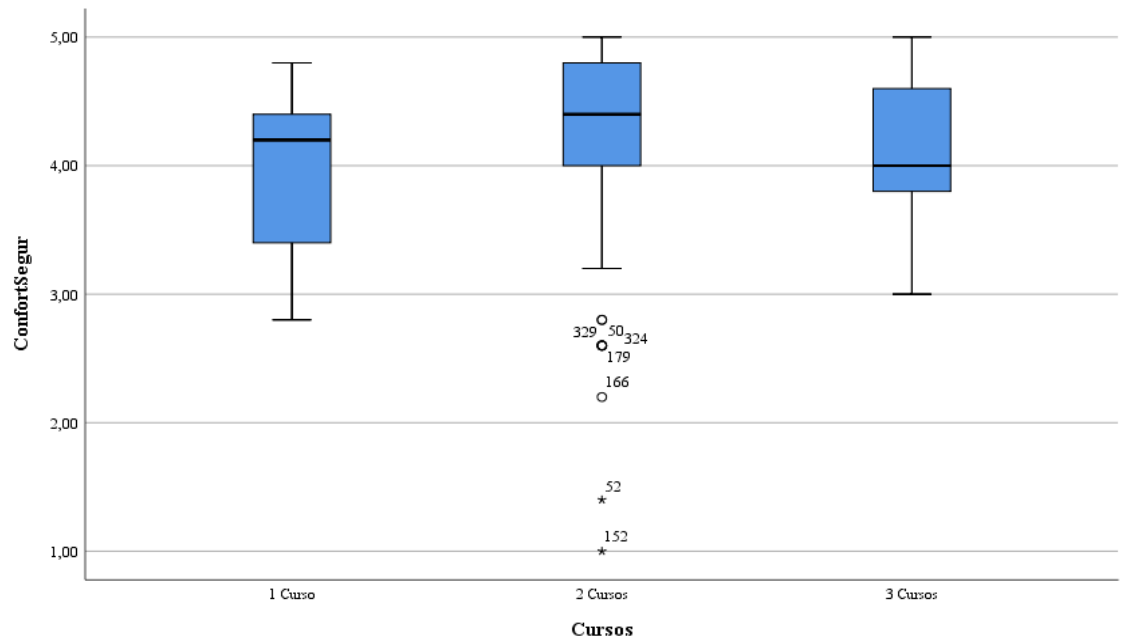

Figura 10. Confort y seguridad emocional en función al número de cursos en el centro Fuente: Elaboración propia.

El factor pertenencia adquiere una distribución muy dispersa en las personas que llevan un único curso, resultando más homogénea en los otros dos grupos. Una vez más, el alumnado con mejores sentimientos de pertenencia es el que lleva dos cursos, con una 
mediana de 4,75, a pesar de la existencia de ocho casos atípicos y tres extremos. Estos sentimientos más positivos implican mayor sentido de pertenencia y orgullo y menores dificultades para asistir al centro y realizar fuera de él lo que desean hacer.

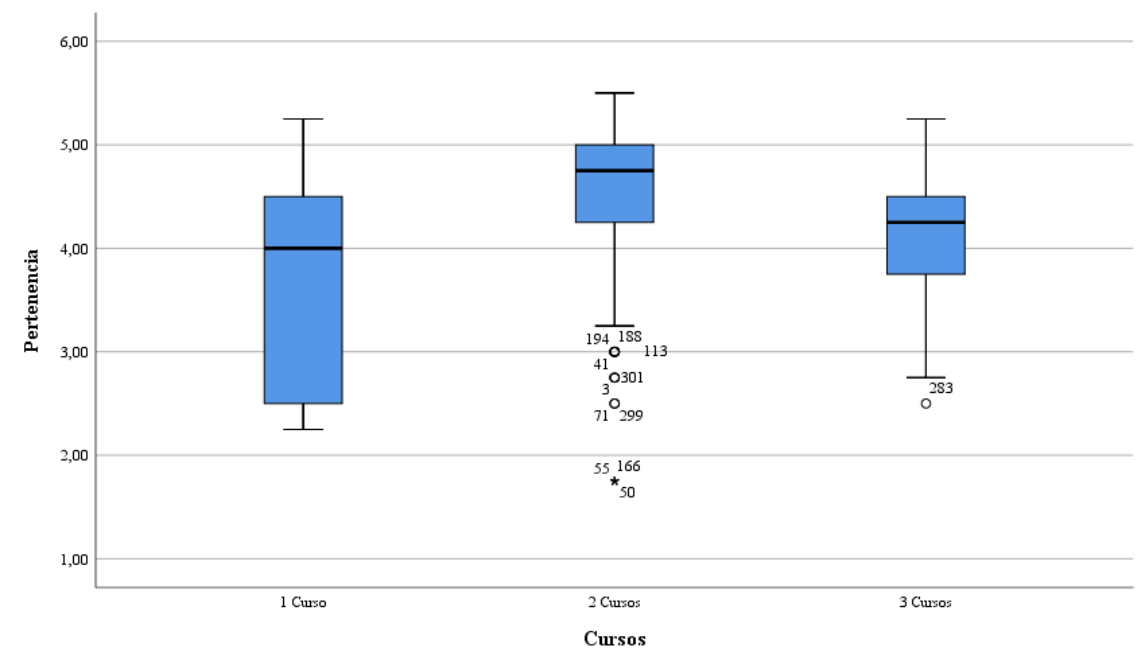

Figura 11. Pertenencia en función al número de cursos en el centro Fuente: Elaboración propia.

Tras este análisis, se aplica la prueba post-hoc de Kruskall-Wallis que permite la comparación de parejas. Posteriormente, se realiza la corrección de Bonferroni ( $p \geq 0,017)$ y se detecta que el único factor significativo para esta variable es la pertenencia, tal y como se recoge en el cuadro 13 .

Cuadro 13. Comparativa entre grupos en función al número de cursos

\begin{tabular}{llcc}
\hline & & SIGNIFICATIVIDAD & SIGNIFICATIVIDAD AJUSTADA \\
\hline 1 curso & 3 cursos & 0,595 & 1 \\
1 curso & 2 cursos & 0,008 & 0,025 \\
3 cursos & 2 cursos & 0,001 & 0,002 \\
\hline
\end{tabular}

Fuente: Elaboración propia.

Se puede establecer la existencia de diferencias significativas entre el alumnado que lleva un curso y el que lleva dos cursos $(0,025)$ y también entre el que lleva dos y tres cursos $(0,002)$. De este modo, los estudiantes que llevan dos cursos tienen una pertenencia mayor que los que llevan un curso, por su reciente llegada al centro, y los que llevan tres cursos, ya que estarían repitiendo el curso en el que se encuentran, lo que puede justificar la falta de pertenencia con el mismo.

\subsubsection{Circunstancia socio-familiar}

En el caso de la existencia o no de circunstancias socio-familiares que supongan una dificultad en el centro, todas las variables han resultado significativas $(\mathrm{p} \leq 0,05)$, como se recoge en el cuadro 14,

Cuadro 14. Grado de significatividad. Cruce de la variable sociodemográfica "circunstancia socio-familiar" con las variables de los factores obtenidos

\begin{tabular}{cccc}
\hline $\begin{array}{c}\text { DINÁMICAS } \\
\text { ESCOLARES }\end{array}$ & $\begin{array}{c}\text { ACTIV. } \\
\text { INDIVIDUAL }\end{array}$ & $\begin{array}{c}\text { CONFORT. } \\
\text { SEGUR. }\end{array}$ & PERTENENCIA \\
\hline 0,016 & 0,046 & 0,002 & 0,000 \\
\hline
\end{tabular}

Fuente: Elaboración propia. 
Para facilitar la comprensión de estos datos, se muestra el cuadro 15 con las medias, medianas y desviación típica obtenidas para cada factor:

Cuadro 15. Número de sujetos, media, desviación típica y mediana en función a la tenencia de circunstancias socio-familiares adversas

\begin{tabular}{|c|c|c|c|c|c|}
\hline $\begin{array}{c}\text { Circunstancia } \\
\text { ADVERSA }\end{array}$ & DESCRIPTIVOS & $\begin{array}{l}\text { DINÁMICAS } \\
\text { ESCOLARES }\end{array}$ & $\begin{array}{l}\text { ACTIVIDADES } \\
\text { INDIVIDUALES }\end{array}$ & $\begin{array}{c}\text { CONFORT } \\
\text { Y } \\
\text { SEGURIDAD }\end{array}$ & Pertenencia \\
\hline \multirow{4}{*}{$\mathrm{Si}$} & $\mathrm{N}$ & 6 & 6 & 6 & 6 \\
\hline & Media & 3,33 & 3,55 & 3,73 & 3,66 \\
\hline & Desv. Tip. & 1,75 & 0,875 & 0,902 & 0,911 \\
\hline & Mediana & 2,71 & 3,50 & 3,90 & 3,50 \\
\hline \multirow{4}{*}{ No } & $\mathrm{N}$ & 78 & 78 & 78 & 78 \\
\hline & Media & 1,93 & 3,12 & 4,30 & 4,46 \\
\hline & Desv. Tip. & 0,628 & 0,857 & 0,578 & 0,766 \\
\hline & Mediana & 1,86 & 3,00 & 4,40 & 4,50 \\
\hline
\end{tabular}

Fuente: Elaboración propia.

Con una mediana de 1,86, las personas sin circunstancias adversas presentan unos mejores sentimientos para el factor dinámicas escolares. Como se puede observar en la figura 12, la dispersión en las respuestas es mayor para las personas con dificultades socio-familiares.

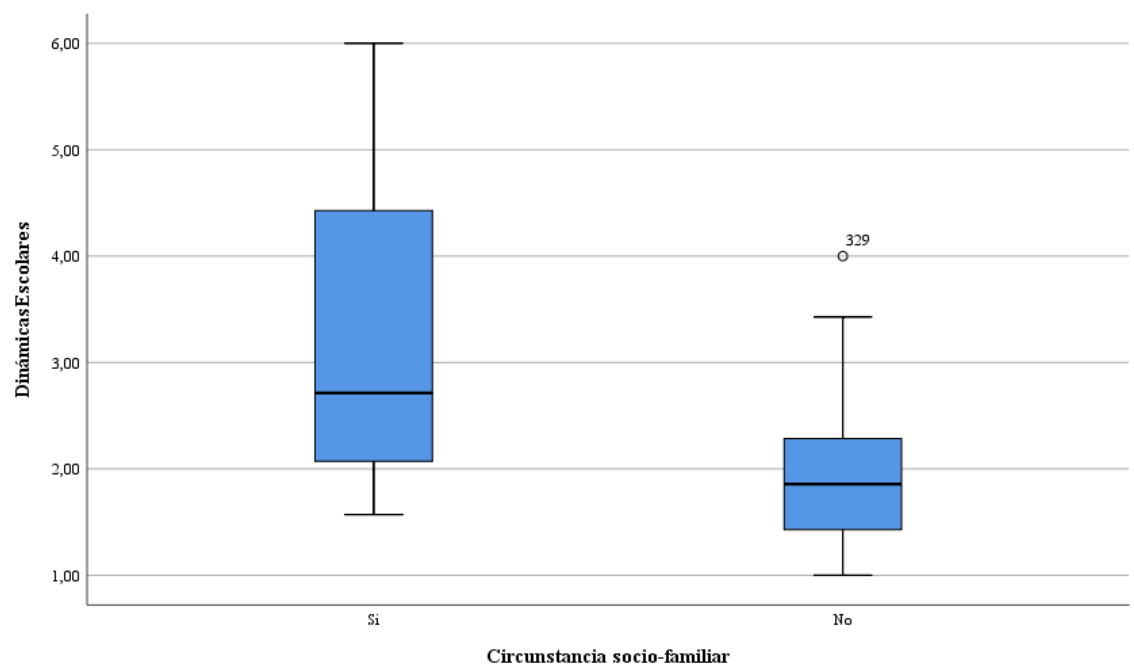

Figura 12. La circunstancia socio-familiar en relación a las dinámicas escolares Fuente: Elaboración propia.

Para la variable actividades académicas de tipo individual, la mediana de las personas que sí tienen dificultades socio-familiares es mayor, por lo que sus sentimientos son peores que los que no las tienen. No obstante, la dispersión es bastante homogénea tal y como muestra la figura 13. 


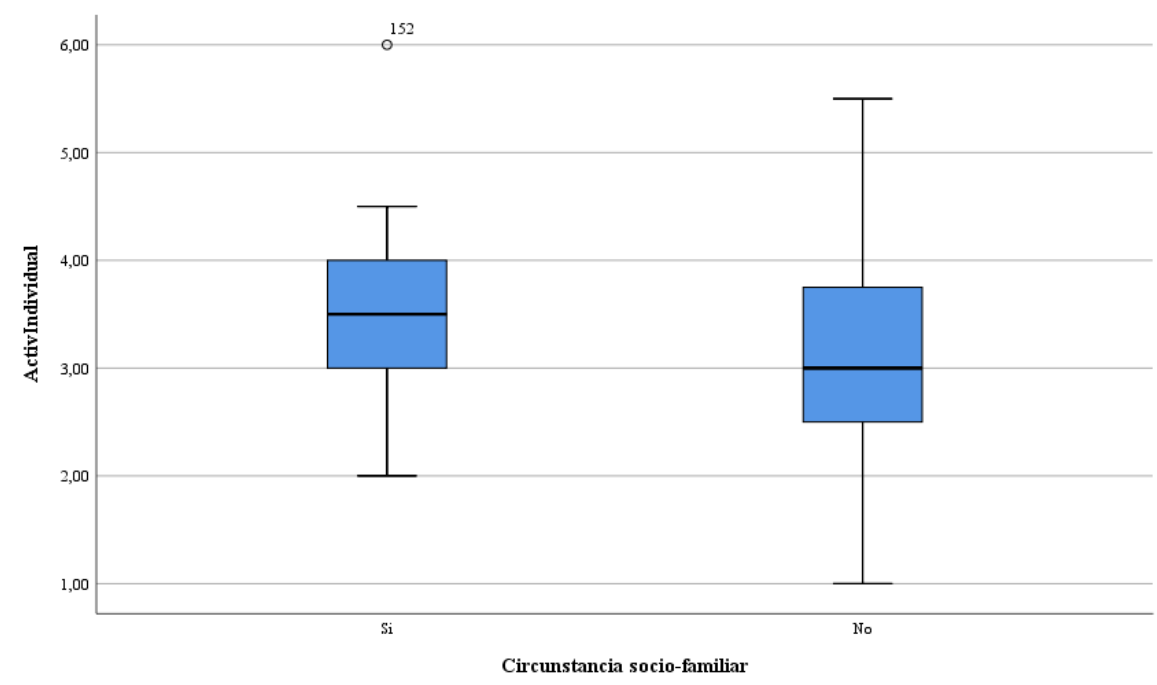

Figura 13. La circunstancia socio-familiar en relación a la actividad individual Fuente: Elaboración propia.

En relación con el confort y la seguridad, las personas sin situaciones complejas se sienten mejor para este factor que las que sí las tienen. La dispersión es homogénea en las respuestas a pesar de existir casos atípicos y extremos, como puede observarse en la figura 14.

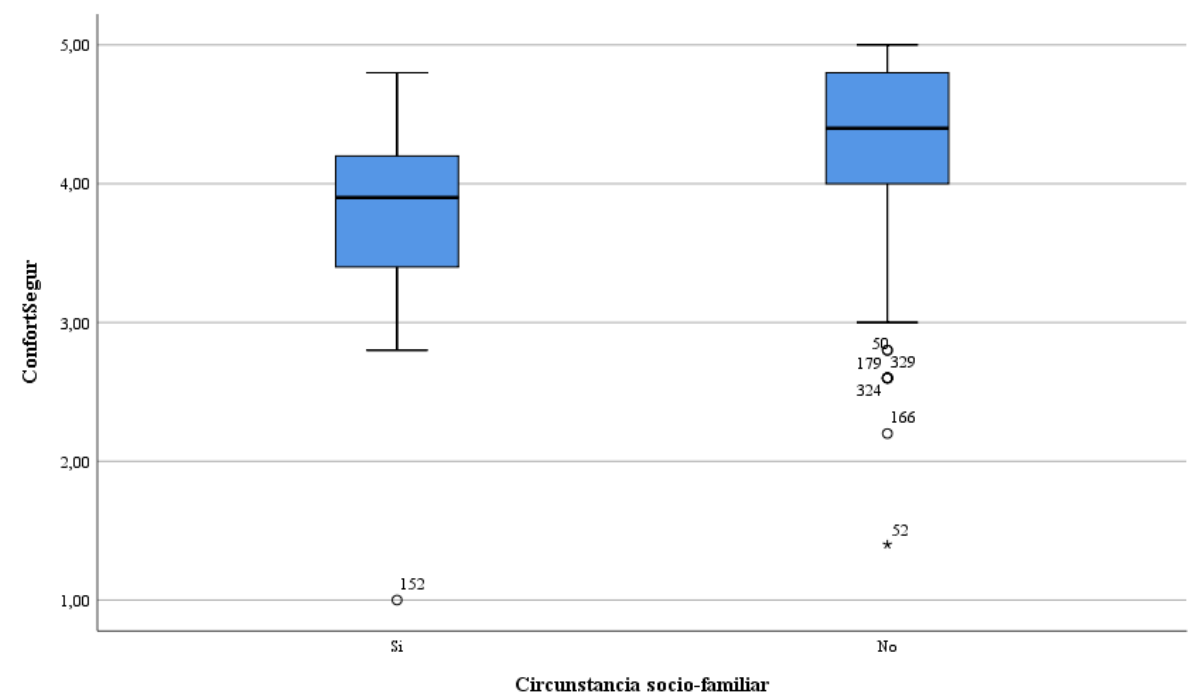

Figura 14. La circunstancia socio-familiar en relación al confort y la seguridad Fuente: Elaboración propia.

En cuanto a la pertenencia, el alumnado sin dificultades socio-familiares experimenta unos sentimientos más positivos hacia las variables de este factor. Asimismo, la dispersión en las respuestas es mayor en el alumnado con dificultades, como muestra la figura 15. 


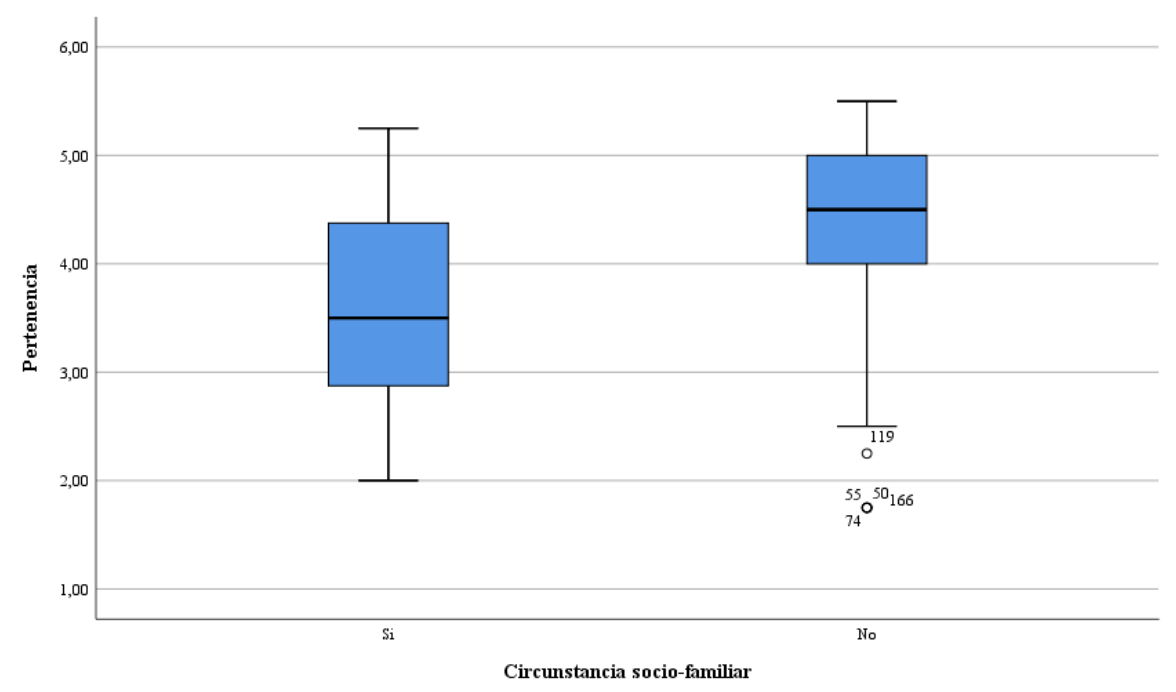

Figura 15. La circunstancia socio-familiar en relación a la pertenencia Fuente: Elaboración propia.

\section{Discusión y conclusiones}

Son múltiples las evidencias de mejora en el terreno educativo, en el que cuestiones como las nuevas metodologías de aprendizaje, la extensión de la tecnología en la educación o una mayor preocupación por la educación inclusiva han ido ganando peso. No obstante, este esfuerzo no parece corresponderse con los altos índices de abandono y fracaso escolar que nos asolan, a pesar de la autonomía pedagógica que los centros educativos tienen sobre este hecho (Domingo y Martos, 2017).

Para paliar y reducir los puntos negros del sistema educativo, es preciso abordar el campo de trabajo de forma directa. Así, el marco de actuación se sitúa en las instituciones, donde docentes, estudiantes y demás personal desarrollan su actividad cotidiana (Amores y Ritacco, 2012).

Es indiscutible que nos encontramos ante un gran reto cuyos cambios tomarán tiempo en ofrecer resultados. En este sentido, no se trata tanto de grandes inversiones como de buenas ideas que favorezcan acciones. Por este motivo, este trabajo presenta una serie de conclusiones a raíz de un estudio exploratorio en el que se pretendía conocer el ámbito para, posteriormente, pensar y aplicar esas "buenas ideas" que dentro del propio centro se pueden desarrollar.

Estas conclusiones sobre el análisis descriptivo se concretan en las a continuación formuladas:

- Existen sentimientos positivos por parte de los estudiantes en los diferentes momentos y lugares de sus centros educativos, a pesar de que las clases siguen siendo las menos valoradas.

- Exámenes y deberes son el talón de Aquiles de nuestros estudiantes, ya que suponen casi el $100 \%$ de la evaluación que se realiza sobre el alumnado. Es por este motivo por el que los sentimientos ante las diferentes formas de trabajo en el aula se encuentran empobrecidos por estos dos condicionantes. 
- En general, existen buenos sentimientos de pertenencia y orgullo con el centro en todos los casos.

En relación con las conclusiones sobre el análisis comparativo, se presentan las siguientes:

- Las personas que se identifican con un género diferente a hombre o mujer adquieren una menor pertenencia que el resto.

- Las personas que siguen la trayectoria normal de número de cursos en el centro, es decir, no son nuevos/as ni están repitiendo, presentan mejores sentimientos para todos los factores estudiados.

- Las personas con dificultades socio-familiares presentan unos sentimientos más pobres para todos los factores estudiados.

A la vista de estas conclusiones, los centros tienen la posibilidad de virar hacia un horizonte más favorable. Entendiendo que las clases son poco valoradas y que los métodos de evaluación son repudiados, una posibilidad sería el trabajo por proyectos, el incremento del trabajo grupal, la conexión de los aprendizajes con la realidad, el fomento de la participación, el trabajo con los grupos que se han destacado como "en riesgo" y, también, favorecer la coordinación, apertura y formación de los profesores/as, así como la observación entre docentes y entre centros, como forma de captar ideas que a otros colegas les están funcionando.

Tras este análisis es importante reconocer este trabajo como un punto de partida de lo que el micro-proyecto supone a nivel general; es decir, este estudio ha permitido conocer y asentar los sentimientos de los estudiantes para después profundizar y trabajar sobre las fortalezas y debilidades de la conexión escolar. El análisis descriptivo y comparativo realizado es de carácter exploratorio, ya que en las siguientes fases del proyecto se trata, de forma cualitativa, de abordar cuestiones particulares que se focalicen en la mejora de los centros a nivel concreto partiendo de las premisas expuestas en el marco teórico.

De esta forma este trabajo explora descriptivamente las variables recogidas en el cuestionario y confirma, mediante el análisis comparativo, el riesgo de ciertos grupos a una menor pertenencia, hecho que puede resultar obvio, pero ha decido asentarse. El hecho de contar con centros de similares características y, por tanto, alumnado homogéneo, impide valorar la extensión de estos resultados a otros contextos. En esta línea, se encuadra este hecho como propuesta de mejora y extensión de este estudio. Asimismo, la traducción y adaptación del cuestionario original también ha sido una limitación.

\section{Agradecimientos}

El presente artículo ha sido desarrollado en el marco del proyecto "Redes de Innovación para la Inclusión Educativa y Social (RIIES)", financiado por el Plan Nacional de Investigación (EDU2015-68617C4-1-R) y dirigido por la profesora Ángeles Parrilla Latas (Universidad de Vigo). Asimismo, este estudio es posibilitado por el contrato predoctoral de la autora para la formación de doctores 2016 del Ministerio de Economía y Competitividad. 


\section{Referencias}

Amores, J. y Ritacco, M. (2012). Prácticas inclusivas ante el riesgo de exclusión socio-educativa. Estructuras de trabajo colaborativo en centros escolares situados en zonas de deprivación social. Revista Internacional de Educación para la Justicia Social, 1(1), 153-178. https://doi.org/10.1037/0022-0663.94.4.795

Anderman, E. (2002). School effects on psychological outcomes during adolescence. Journal of Educational Psychology, 94(4), 795-809.

Appleton, J. J., Christenson, S. L. y Furlong, M. J. (2008). Student engagement with school: Critical conceptual and methodological issues of the construct. Journal of School Psychology, 45, 369386. https://doi.org/10.1002/pits.20303

Boza, J. Pérez, J. y de León, J. (2016). Introducción a las técnicas de muestreo. Pirámide.

Baumeister, R. y Leary, M. (1995). The need to belong: Desire for interpersonal attachments as a fundamental human motivation. Psychological Bulletin, $117(3)$, 487-529.

https://doi.org/10.1037/0033-2909.117.3.497

Bond, L., Butler, H., Thomas, L., Carlin, J., Glover, S., Bowes, G. y Patton, G. (2007). Social and school connectedness in early secondary school as predictors of late teenage substance use, mental health, and academic outcomes. Journal of Adolescent Health, 40, 9-18.

https://doi.org/10.1016/j.jadohealth.2006.10.013

Canales. D. y Peña, L. (2014). Factores que impactan en el sentido de pertenencia en la escuela: Dibujos y relatos de estudiantes de séptimo básico en cuatro escuelas municipales. Pontificia Universidad Católica de Valparaíso.

Domingo, J. y Martos, J. (2017). Prevención del fracaso escolar en educación secundaria en Andalucía desde la voz del profesorado. Estudio de caso. Revista Internacional de Educación para la Justicia Social, 6(1), 329-347. https://doi.org/10.15366/riejs2017.6.1.019

Eisner, C. (2001). Making the grade: A report on SAT I results in the nation's urban schools. Council of the Great City Schools.

Fielding, M. (2011). Beyond student voice: Patterns of partnership and the demands of deep democracy. Revista de Educación, 359, 45-65.

Finn, J. D. (1989). Withdrawing from school. Review of Educational Research, 59, 117-142. https://doi.org/10.3102/00346543059002117

Freeman, T., Hughes, H. y Anderman, L. H. (abril, 2001). Changes in students' school belonging in rural and urban middle schools. Poster presented at the Annual Meeting of the American Educational Research Association, Seattle.

García, J. (2015). El fracaso escolar desde la perspectiva de la exclusión educativa. El curriculum prescrito del PDC y del PCPI en la Comunidad Valenciana. Universitat de València.

Goodenow, C. (1993). Classroom belonging among early adolescent students: Relationships to motivation and achievement. Journal of Early Adolescence, 13(1), 21-43.

https://doi.org/10.1177/0272431693013001002

González González, M. T. (2015). Los centros escolares y su contribución a paliar el desenganche y el abandono escolar. Profesorado, Revista de Currículum y Formación del Profesorado, 19(3), 158-176.

Hancock, K. J. y Zubrick. S. (2015). Children and young people at risk of disengagement from school. Telethon Kids Institute. 
Hargreaves, A., Earl, L. y Ryan, J. (1996). Schooling change: Reinventing education for early adolescents. Falmer.

Hernández, R., Fernández, C. y Baptista, P. (2010). Metodología de la investigación. McGrawn Hill.

Loukas, A., Suzuki, R. y Horton, K. (2006). Examining school connectedness as a mediator of school climate effects. Journal of Research on Adolescence, $16(3), 491-502$. https://doi.org/10.1111/j.1532-7795.2006.00504.x

Luckner, A. y Pianta, R. (2011). Teacher-student interaction in fifth grade classrooms: Relation with children's peer behavior. Journal of Applied Developmental Psychology, 32(5), 257-266. https://doi.org/10.1016/j.appdev.2011.02.010

McMillan, D. W. y Chavis, D. M. (1986). Sense of community: A definition and theory. Journal of Community Psychology, 14, 6-23. https://doi.org/10.1002/1520-6629(198601)14:1<6::AIDJCOP2290140103>3.0.CO;2-I

Mahatmya, D., Lohman, B., Matjasko, J. y Feldman, A. (2012). Engagement across developmental periods. En S. L. Chistenson, A. L. Reschly y C. Wylie (Eds.), Handbook of research on student engagement (pp. 45-63). Springer. https://doi.org/10.1007/978-1-4614-2018-7_3

Martin, A. J. (2007). Examining a multidimensional model of student motivation and engagement using a construct validation approach. Bristish Journal of Education Psichology, 77(2), 413440. https://doi.org/10.1348/000709906X118036

Porter, J., Daniels, H., Martin, S., Hacker, J., Feiler, A. y Georgeson, J. (2010). Testing of disability identification tool for schools. University of Bath Department of Education.

Resnick, M. D., Bearman, P. S., Blum, R. W., Bauman, K. E., Harris, K. M., Jones, J. .., y Udry, J. R. (1997). Protecting adolescents from harm. Findings from the national longitudinal study on adolescent health. Journal of the American Medical Association, 278(10), 823-832. https://doi.org/10.1001/jama.1997.03550100049038

Shochet, I. y Smith, C. (2014). A prospective study investigating the links among classroom environment, school connectedness and depressive symptoms in adolescents. Psychology in the Schools, 51(5), 480-491. https://doi.org/10.1002/pits.21759

Skinner, E., Furrer, C., Marchand, G. y Kinderman, T. (2008). Engagement and disaffection in the classroom: Part of a larger motivational dynamic? Journal of Educational Psychology, 100, 765-781. https://doi.org/10.1037/a0012840

Terigi, F. (2014). Trayectorias escolares e inclusión educativa. Del enfoque individual al desafío para las políticas educativas. En A. Marchesi, R. Blanco, y L. Hernández (Coords.), Avances y desafíos de la educación inclusiva en Iberoamérica (pp. 71-87). OEI.

Thompson, D., Iachan, R., Overpeck, M., Ross, G. y Gross, L. (2006). School connectedness in the health behaviour in school-aged children study: The role of student, school, and school neighborhood characteristics. Journal of School Health, 76(7), 379-386.

https://doi.org/10.1111/j.1746-1561.2006.00129.x

\section{Breve CV de la autora}

\section{Isabel Fernández Menor}

Graduada en Pedagogía por la Facultad de Ciencias de la Educación de Santiago de Compostela y Máster en Dificultades de Aprendizaje y Procesos Cognitivos en la Universidad de Vigo. En el año 2016 inicia sus estudios de doctorado en la Universidad de Vigo (Campus Pontevedra) en el programa de Equidad e Innovación en Educación y 
obtiene un contrato predoctoral del Ministerio de Economía y Competitividad para la realización de la tesis doctoral. Su tesis versa sobre la pertenencia y los procesos de enganche y desenganche escolar desde una perspectiva inclusiva en educación secundaria. Desde el año 2017 imparte docencia en los Grados de Maestro en Educación Primaria e Infantil de la Universidad de Vigo en materias como Educación Inclusiva, Tutoría y Orientación con las Familias y Nuevas Tecnologías aplicadas a la Educación Primaria. ORCID ID: https://orcid.org/o000-0003-2873-6952. Email: isfernandez@uvigo.es 